\title{
Origin of oligodendrocytes in mammalian forebrains: a revised perspective
}

\author{
Masae Naruse $^{1,2} \cdot$ Yasuki Ishizaki $^{2} \cdot$ Kazuhiro Ikenaka $^{1} \cdot$ Aoi Tanaka $^{3}$. \\ Seiji Hitoshi ${ }^{1,3}$
}

Received: 15 April 2016/ Accepted: 16 August 2016/Published online: 29 August 2016

(c) The Physiological Society of Japan and Springer Japan 2016

\begin{abstract}
Oligodendrocyte precursor cells (OPCs) appear in the late embryonic brain, mature into oligodendrocytes (OLs), and form myelin in the postnatal brain. It has been proposed that early born OPCs derived from the ventral forebrain are eliminated postnatally and late-born OLs predominate in the adult mouse cortex. However, the temporal and regional niche for cortical OL generation, which persists throughout life in adult mammals, remains to be determined. Our recent study provides new insight into self-renewing and multipotent neural stem cells (NSCs). Our results, together with previous studies, suggest that NSCs at the dorsoventral boundary are uniquely specialized to produce myelin-forming OLs in the cortex during a restricted temporal window. These findings may help identify transcription factors or gene expression patterns which confer neural precursors with the characteristic ability of dorsoventral boundary NSCs to differentiate into OLs, and facilitate the development of new strategies for regenerative medicine of the damaged brain.
\end{abstract}

Keywords Cortical oligodendrocyte $\cdot$ Dorsoventral boundary $\cdot$ Neural stem cells $\cdot$ Wnts $\cdot$ FGF

Seiji Hitoshi

shitoshi-tky@umin.ac.jp

1 Division of Neurobiology and Bioinformatics, National Institute for Physiological Sciences, Okazaki, Japan

2 Department of Molecular and Cellular Neurobiology, Gunma University Graduate School of Medicine, Maebashi, Japan

3 Department of Integrative Physiology, Shiga University of Medical Science, Otsu, Shiga, Japan ....as the name "interfascicular glia" indicates only the principal feature of its constitutive elements, we have adopted the term "oligodendroglia"...Unfortunately, we have not been able to observe the very first stages of genesis of the oligodendroglia... by Pío Del Río-Hortega [1]

\section{Introduction}

Myelination of axons by mature cortical oligodendrocytes (OLs) starts during late embryonic stages and persists long into adulthood in the mammalian brain [2]. This led to the notion that cortical OLs are "generated" in the postnatal brain for a fairly extended period—but, "generated" from what? All OLs are considered to be progeny of neural stem cells (NSCs), which are present not only in embryonic brains but also in specific neurogenic regions of the adult brain, and produce new neurons in the olfactory bulb and dentate gyrus of the hippocampus $[3,4]$. NSCs proliferate during early embryonic stages, produce neurons during middle embryonic stages, then generate OLs and astrocytes during late embryonic to postnatal stages (Fig. 1). Oligodendrocyte precursor cells (OPCs) represent an intermediate stage in oligodendrogenesis but also persist in the adult cortex as Olig2 ${ }^{+} /$ $\mathrm{NG}^{+}$cells $[5,6]$. OPCs vigorously proliferate just before terminal differentiation into mature OLs [7]; this makes birthdating studies of OLs extremely difficult because common labels using nucleotide analogues, such as BrdU, would be diluted to below detection limits. Therefore, the temporal window in which OL-lineage cells are segregated from self-renewing and multipotent NSCs remains elusive. 
Fig. 1 Neural stem cell (NSC) proliferation and differentiation in the embryonic mouse brain. Neural stem cells (shown in yellow), or radial glial cells, produce neurons (green) in the middle embryonic stage, and then oligodendrocytes (blue) and astrocytes (purple) in the late embryonic stage

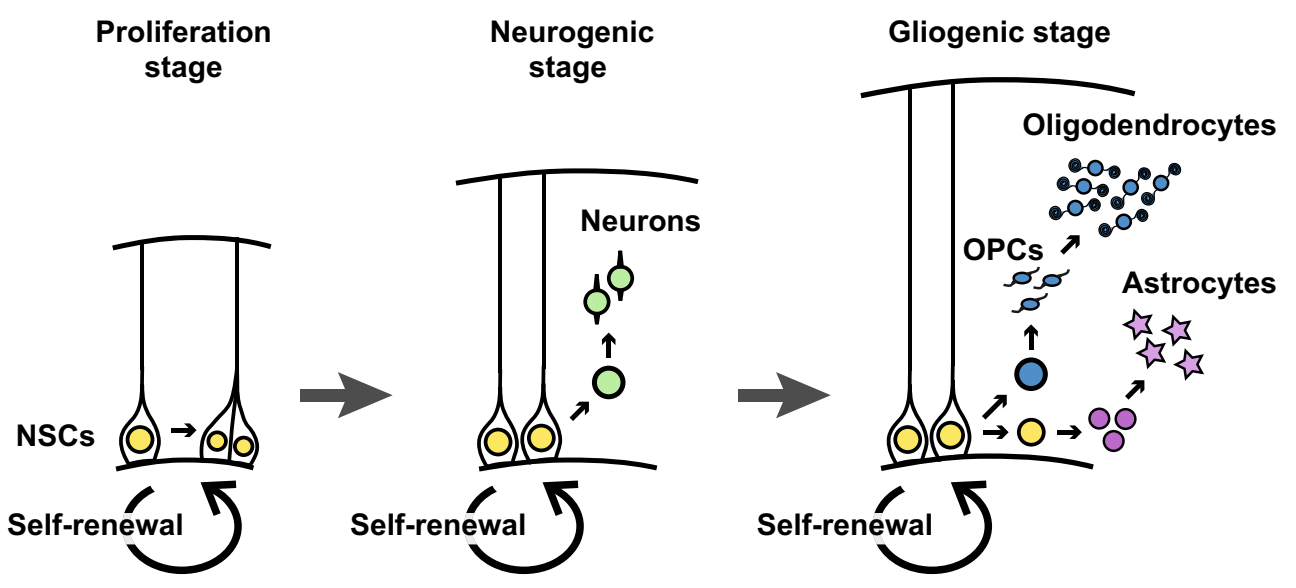

\section{Temporal window of oligodendrocyte generation}

\section{Lineage tracing system}

Self-renewing NSCs in the developing brain produce multipotent, non-self-renewing neural progenitor cells (NPCs). Once NSCs exit the self-renewing cycle, differentiation appears uni-directional into lineage-committed progeny $[8,9]$. To examine when NSCs exit the self-renewing stem cell population and begin to differentiate into OPCs, we established a system to separately label the selfrenewing NSC population and non-self-renewing NPC/ OPC population at various time points [10]. We utilized a Cre-LoxP system in which Cre-Estrogen Receptor (ER) fusion protein-expressing cells can be permanently labeled at specific time points by injection with 4-hydroxytamoxifen (4-OHT) to induce recombination and expression of a reporter gene, such as GFP in the Z/EG reporter mouse line. In Nestin-CreER;Z/EG double transgenic mice, CreER expression is driven by the Nestin promoter/enhancer to reflect the endogenous Nestin gene expression pattern that is restricted to the VZ/SVZ, where self-renewing NSCs reside. If administration of 4-OHT labels self-renewing neural stem cells, labeled cells should remain in the VZ/SVZ during later developmental stages and yield $\mathrm{GFP}^{+}$neurospheres in in vitro colony-forming NSC cultures (a neurosphere assay). Indeed, 3 days after 4-OHT administration to E11.5 Nestin-CreER;Z/EG embryos, $\mathrm{GFP}^{+}$cells were found in the VZ/SVZ, some of which formed $\mathrm{GFP}^{+}$neurospheres in the neurosphere assay (Fig. 2a).

Olig2, which is considered an OL-lineage cell marker, is expressed in the VZ/SVZ of embryonic brains in a pattern very similar to that of Nestin. In addition to OLs, Olig2lineage cells differentiate into neurons and astrocytes [10-13], suggesting that it is expressed in multipotent neural stem/progenitor cells (or collectively, neural precursor cells). However, Olig2-lineage cells did not remain in the VZ/SVZ but migrated out to differentiate and never formed $\mathrm{GFP}^{+}$neurospheres (Fig. 2a), in sharp contrast to Nestin-lineage cells. This difference in Nestin- and Olig2lineage cells makes it possible to differentially label multipotent and self-renewing NSCs and their non-self-renewing and OL-lineage progeny (Fig. $2 b$ ).

\section{Restricted period of OL lineage cell differentiation}

We administered 4-OHT to Nestin-CreER;Z/EG and Olig2 ${ }^{\mathrm{CreER}} ; \mathrm{Z} / \mathrm{EG}$ embryos at E12.5, E16.5, or P10 and examined the presence or absence of $\mathrm{GFP}^{+}$cells in the cortex at P30 (Fig. 2c). $\mathrm{GFP}^{+}$cortical OLs were observed in Nestin-CreER mice treated with 4-OHT at E12.5 or E16.5 but not at P10, demonstrating that the generation of OL-lineage cells from the NSC population has ceased by $\mathrm{P} 10$. On the other hand, while there are many $\mathrm{GFP}^{+} \mathrm{OLs}$ in the cortex of $\mathrm{Olig} 2^{\mathrm{CreER}}$ mice treated with 4-OHT at E16.5 or $\mathrm{P} 10$, no $\mathrm{GFP}^{+}$cortical OLs were detected in those treated at E12.5, suggesting that NPCs, which are fated to become cortical OLs in the adult brain, have yet to be generated from NSCs at E12.5. These results suggest that neural progenitor cells, which subsequently differentiate into cortical OLs in the adult brain, are derived from selfrenewing NSCs during a period just prior to E16.5 through P10.

\section{Oligodendrocyte generating niche in the developing brain}

\section{Patterning of the central nervous system}

In the developing central nervous system (CNS), morphogens such as bone morphogenetic proteins (BMPs), Wnts, sonic hedgehog (Shh), and fibroblast growth factors (FGFs) form concentration gradients along the dorsoventral and anteroposterior axes, and contribute to 
Fig. 2 Lineage tracing using Cre recombinase

transgenic/knock-in mice. a Nestin-CreER;Z/EG and Olig $2^{\text {CreER }}$;Z/EG embryos were labeled by injection of $2 \mathrm{mg}$ 4-hydroxytamoxifen (4OHT) intraperitoneally into the dam at E11.5, then cells from the germinal zone of the ganglionic eminence were used in a colony-forming neurosphere assay at E14.5. Neurospheres with or without GFP expression are indicated by arrows or arrowheads, respectively. Scale bars $200 \mu \mathrm{m}$. b Schematic diagram of Nestin- and Olig2lineage progeny (cells shown in red and blue, respectively) of neural precursor cells labeled by injection with 4OHT (shown as a vertical green line). c NestinCreER;Z/EG and Olig2 $2^{\text {CreER; }}$ $\mathrm{Z} / \mathrm{EG}$ mice were labeled at the indicated times and brains were analyzed at $\mathrm{P} 30$. $\mathrm{GFP}^{+}$cells in the cortex were classified as oligodendrocytes $(\mathrm{O})$, astrocytes $(\mathrm{A})$, or neurons $(\mathrm{N})$ based on morphology, and the number of cells from each type were counted (modified from Naruse et al. [10])
A

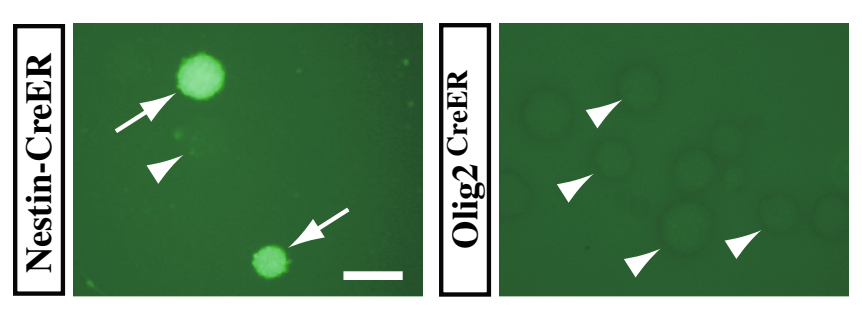

B

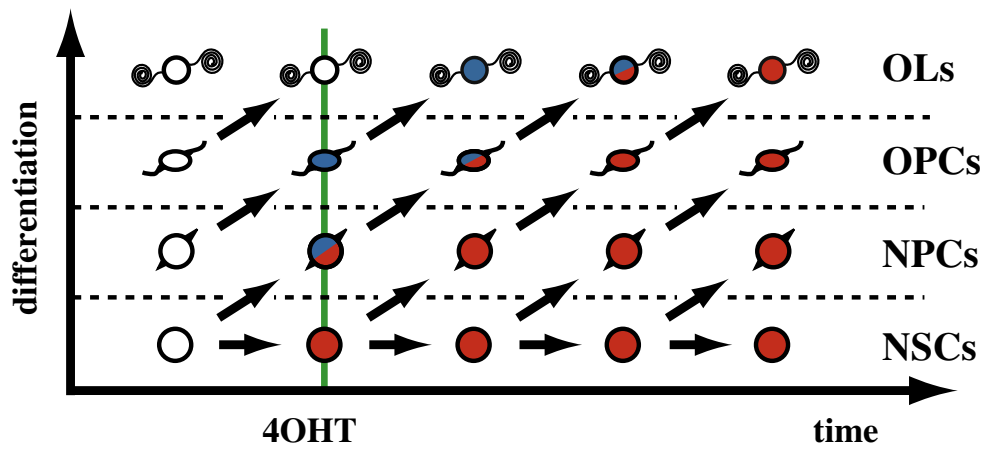

C
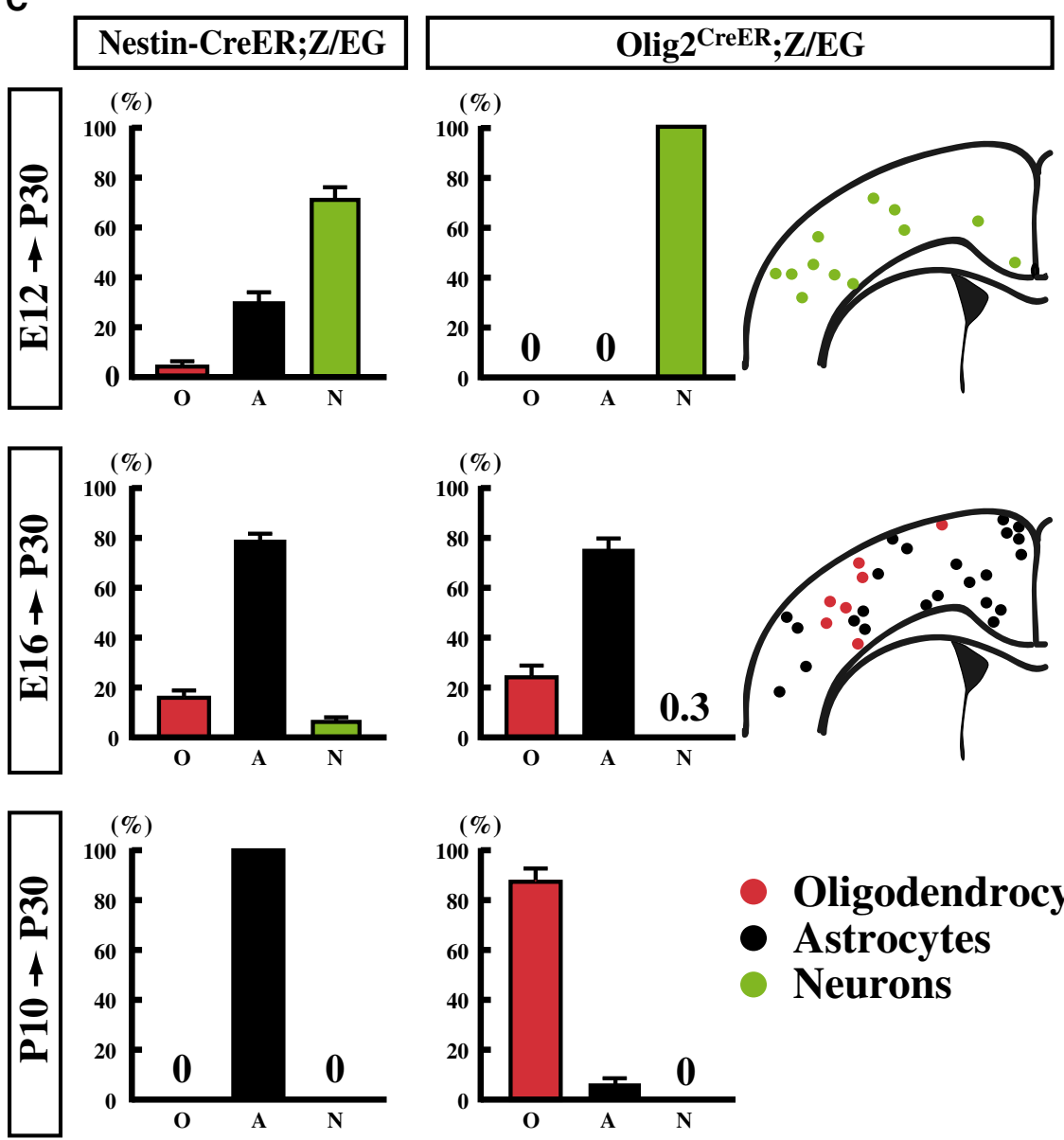

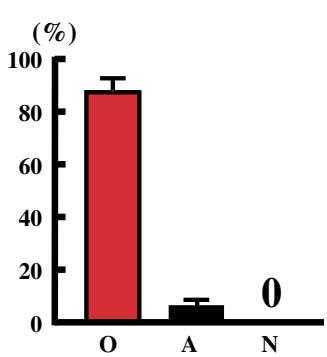

the developmental patterning and regional differentiation of NSCs [14, 15] (Fig. 3). BMPs and Wnts are secreted from the roof plate of the spinal cord and the cortical hem of the telencephalon, and contribute to dorsal patterning [15-19]. Conversely, Shh is secreted from the floor plate and notochord of the spinal cord, and from the ventral 


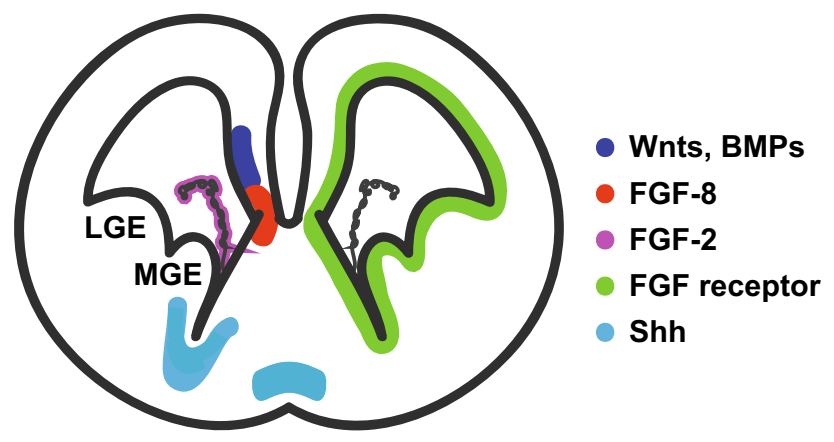

Fig. 3 Distribution of morphogens, which may influence oligodendrogenesis in the embryonic brain, and FGF receptors is shown in a scheme of the coronal section of E14.5 mouse brain. LGE and MGE mean lateral and medial ganglionic eminence, respectively

regions of the telencephalon $[20,21]$, leading to the establishment of ventral patterning [22-25]. While NSCs are regionally specified along the dorsoventral and anteroposterior axes [26, 27], neurons and astrocytes are produced from most, if not all, regions of the VZ/SVZ throughout the entire CNS. In contrast, oligodendrocyte generation takes place in a few restricted regions, although the specific origins of OLs in the CNS have been controversial for years. Recent studies, including ours, have provided a new perspective on this issue; in this section, the current views on the source of OLs in both the spinal cord and forebrain will be discussed.

\section{Traditional perspectives on oligodendrocyte generation-spinal cord}

The developmental origin of OLs has been extensively investigated in the spinal cord through analysis of several knockout mouse lines. Around E12, OL-lineage cells expressing platelet-derived growth factor alpha-receptor (PDGFR $\alpha$ ), an established marker for OPCs, are derived from the motor neuron progenitor (pMN) domain (Fig. 4a). The pMN domain harbors specialized neural precursor cells, which first give rise to motoneurons, then switch their fate to produce OPCs [28]. Shh is secreted from the floor plate and notochord, and is indispensable for ventral patterning of the developing spinal cord [29]. Mouse embryos deficient for Shh or both Nkx6.1 and Nkx6.2, homeodomain transcription factors expressed under the influence of Shh, completely lack Olig2 ${ }^{+}$OPCs in the early stage spinal cord [30, 31]. Olig2, a basic helix-loop-helix (bHLH) transcription factor, is expressed in NPCs of the pMN domain and plays an essential role in the development of motoneurons and OPCs [32-34]. This role was demonstrated in studies of Olig2-deficient mice, which were found to be devoid of motoneurons and OPCs in the spinal cord and die shortly after birth $[35,36]$. It was recently reported that phosphorylation of the Olig2 bHLH
A

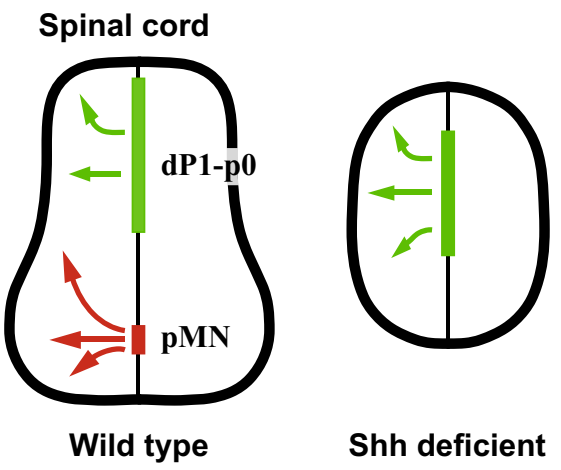

B Telenchepharon

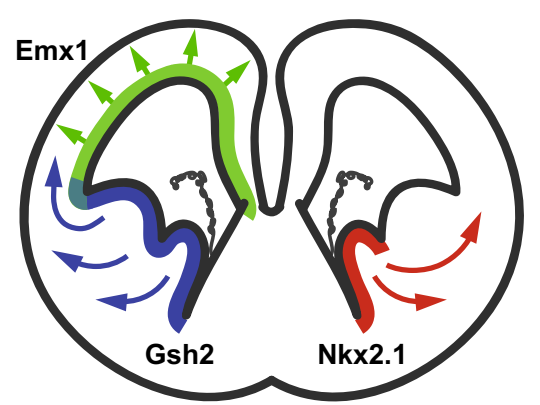

Fig. 4 Origins of cortical oligodendrocytes in the spinal cord (a) and brain (b). a There are at least two sources of OL-lineage cell generation in the embryonic spinal cord: the $\mathrm{pMN}$ domain and more dorsal region. Oligodendrogenesis from the pMN domain is dependent on Shh and disrupted in Shh-deficient mice, whereas dorsal oligodendrogenesis remains in such mice. b A revised model of oligodendrogenesis in the forebrain showing that three sequential waves of OPC generation from neural precursors in different regions: the $\mathrm{Nkx} 2.1^{+}$medial ganglionic eminence (MGE) starting at E12.5, the Gsh2 ${ }^{+}$lateral-to-caudal GE starting at E15.5, and the Emx1 ${ }^{+}$ cortical ventricular zone starting around birth

domain is critical for motoneuron production, and dephosphorylation of Olig2 alters its binding partners and induces OPC generation from the pMN domain [37]. This suggests that OL-lineage cells derived from the pMN domain are generated under strong influence from Shh and the subsequent action of Nkx6 and Olig2.

Interestingly, some OPCs appear in the dorsal portion of the spinal cord in Shh- or Nkx6.1/6.2-deficient embryos after E15 [30], suggesting the presence of an Shh-independent source for OPC production during later embryonic stages. This idea is also supported by data obtained from wild-type embryos in studies using the Cre/loxP system. For example, lineage tracing using Cre recombination under the control of the promoter for the $D b x l$ gene, a homeobox transcription factor expressed in the $\mathrm{p} 0$ and $\mathrm{dP} 6$ domains of the developing spinal cord, or the promoter for the Gsh2 and Msx3 genes, expressed in even more dorsal spinal cord, shows that OPCs are also derived from the dorsal region $[38,39]$. These findings suggest that there are at least two sources of OL-lineage cell generation in the 
embryonic spinal cord: one dependent and the other independent of Shh.

\section{Traditional perspectives on oligodendrocyte generation-forebrain}

The sequential appearance of OPCs, first in the ventral region under the influence of Shh, followed by their presence in the dorsal region independent of Shh, also occurs in the telencephalon. OPCs in the telencephalon first appear in the VZ/SVZ of the ventral region from the medial ganglionic eminence (MGE) to the anterior entopeduncular area (AEP) that is defined by the expression of the $G b x 2$ homeobox gene [40], around E12 [41, 42]. The generation of OPCs in these regions is induced by Shh signaling, which was demonstrated by analyzing Nkx2.1-deficient mouse embryos [41]. Nkx2.1 is a homeodomain transcription factor and critical inducer of Shh in the telencephalon. Deletion of Nkx2.1 leads to the loss of Shh expression in cells surrounding the VZ from the MGE to the AEP, and OPC generation during early embryonic stages. Furthermore, in the Nkx2.1-deficient embryos, OPCs emerge and spread out from the GE to the cortex after E16, suggesting that Shh-independent OPCs also exist in the telencephalon [43]. Interestingly, analysis of mice deficient for Mash1, another bHLH transcription factor, indicated that Mash1 is required for the generation of the early population of OPCs in the ventral forebrain around E12, but not for the later population of OPCs [44]. Thus, two or more populations of OL-lineage cells may exist in the adult mammalian brain, one derived from the ventral forebrain under the influence of Shh and the other(s) from the dorsal forebrain independent of Shh activity.

\section{Revised perspective on oligodendrocyte generation-forebrain}

A lineage-tracing study using Cre-transgenic mice supports the above hypotheses and suggests three waves of OPC generation (Fig. 4b) [45]. The earliest wave originates from the ventral forebrain as indicated above in the traditional point of view, in which OPCs are derived from Nkx2.1-lineage cells of the MGE and AEP. MGE/AEPderived OPCs migrate tangentially into the cortex where they mature and contribute to a subpopulation of myelinating OLs in the adult cortex [46]. Interestingly, most MGE/AEP-derived OLs in the cortex seem to be eliminated postnatally although they survive in the ventral portion of the adult brain [45]. The second wave of OPC generation starts from the lateral ganglionic eminence (LGE); these are labeled in Gsh2-Cre transgenic mice because Gsh2 is expressed in the VZ/SVZ of the LGE and MGE in the developing brain. The third and final wave of
OPCs are derived from the dorsal neural precursors which express Emxl, a dorsal forebrain specific homeobox gene, as shown by the analysis of Emxl-Cre mice. These results demonstrate that most, if not all, of the OLs in the adult cortex are derived from the $E m x I^{+}$dorsal forebrain and $G s h 2^{+}$LGE [45].

\section{Our model of oligodendrocyte generation}

We have reported that dorsal neural precursor cells never contribute to cortical OLs in the adult brain based on an in utero electroporation study using the Cre/loxP system $[10,46]$. This appears to be inconsistent with a recent paper showing that Emxl-lineage OLs predominate in the adult cortex [45]. To resolve this apparent discrepancy, we performed a detailed examination of the origins of cortical OLs and found that neural precursor cells in the lateral dorsoventral boundary that express both Emxl and Gsh2 are a source of adult cortical OLs, whereas those in the dorsal forebrain, excluding the boundary region, only produce neurons and astrocytes (Fig. 5a, b). Thus, the Emx1- and Gsh2-lineage OLs in the adult cortex shown by the preceding study [45] might originate from the Emx1/Gsh2-expressing boundary region. Surprisingly, neural precursor cells in the cortico-striatal (lateral) as well as cortico-septal (medial) boundary regions differentiate into cortical OLs, suggesting that both the lateral and medial dorsoventral boundary regions represent a specialized niche for the generation of cortical OLs (Fig. 6a) [10]. In contrast, OLs in the dorsal-most cortex were never labeled by in utero electroporation in our study, suggesting the possibility of yet another source of cortical OLs in addition to the lateral and medial boundary regions (Fig. 6b).

\section{Roles for Wnt signaling in the generation of cortical OLs}

Oligodendrogenesis is under the influence of several extrinsic factors, among which the Wnt family of proteins plays a major role in suppressing the generation of OPCs from neural precursors [47-49]. Given that Wnt signaling is activated in the dorsomedial portion of the forebrain, it is possible that dorsal neural precursor cells are suppressed by Wnts to differentiate into OL-lineage cells. Inhibition of Wnt signaling by electroporation of $D k k$, an inhibitor of Wnt signals, or a constitutively active form of $\beta$-catenin, results in the generation of a substantial number of OLs from dorsal neural precursor cells (Fig. 5c) [10, 48]. Thus, dorsal neural precursor cells retain their oligodendrogenic capability but are inhibited by Wnts and other extrinsic factors. 
A
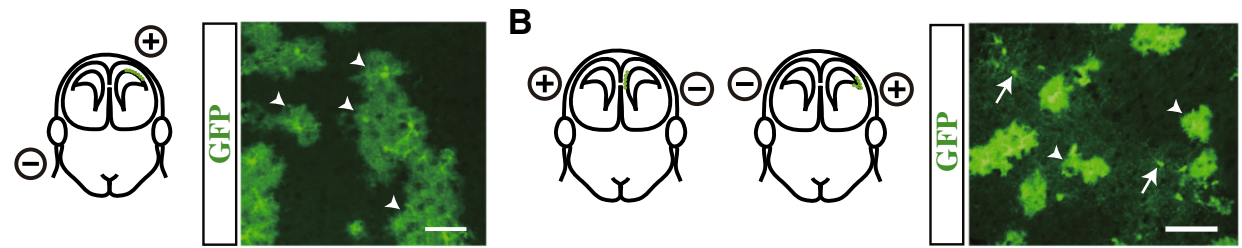

C

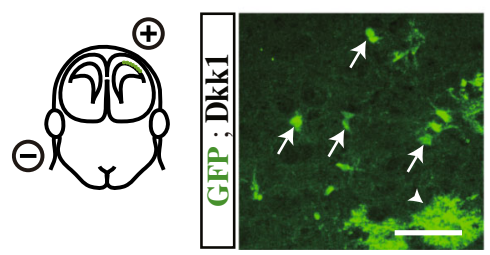

Fig. 5 In utero electroporation. Neural precursor cells in the dorsal cortex (a) or lateral and medial dorsoventral boundary (b) were labeled by electroporating E15.5 Z/EG reporter mouse embryos with a Cre recombinase expression plasmid. $\mathrm{GFP}^{+}$cells were traced in coronal sections of P30 mouse brains and classified as

\section{A}
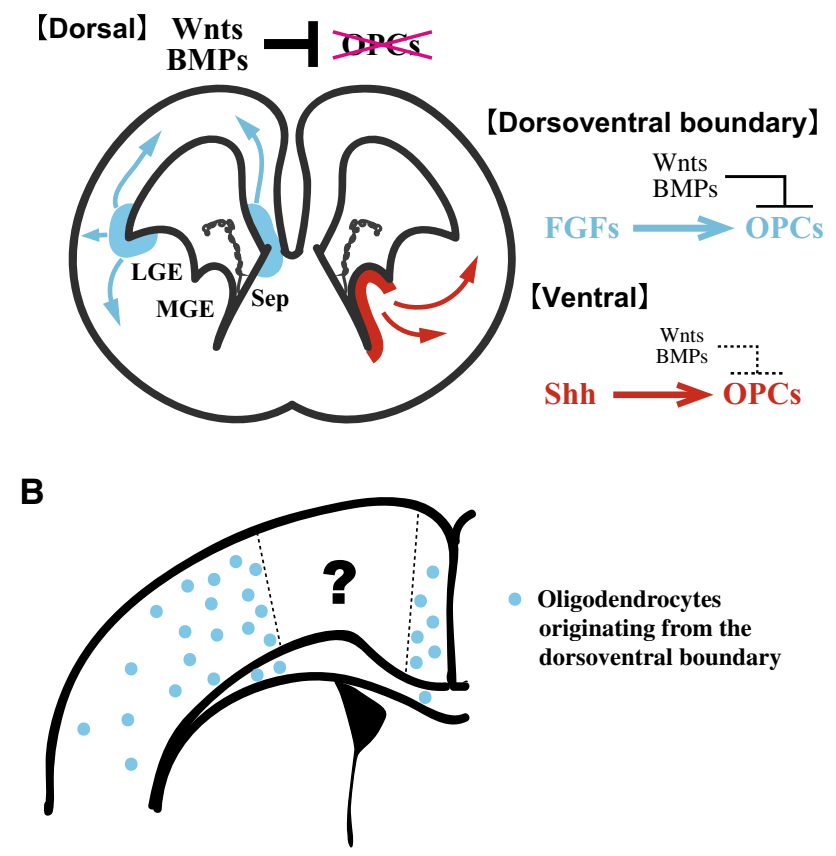

Fig. 6 Proposed model of cortical oligodendrogenesis. a Ventral oligodendrogenesis is dependent on Shh signaling. Shh-independent generation of oligodendrocyte precursor cells (OPCs) occurs at the lateral and medial dorsoventral boundary, where FGF signaling may overcome the suppression of oligodendrogenesis by Wnt signaling. b The origins of the dorsal-most portion of the adult cortex remain to be determined

\section{Roles for FGF signaling in the generation of cortical OLs}

Wnt proteins are secreted from the cortical hem in the developing brain and distributed in a gradient, with higher concentrations dorsomedially and lower concentrations

oligodendrocytes (arrows), astrocytes (arrowheads), or neurons (not shown) based on morphology. c Suppression of Wnt signaling by the expression of $D k k$ caused neural precursor cells in the dorsal cortex to also differentiate into oligodendrocytes (arrows). Scale bars $50 \mu \mathrm{m}$ (modified from Naruse et al. [10])

ventrolaterally [52]. The strong oligodendrogenesis-suppressing Wnt signal that is active in the dorsomedial portion of the embryonic forebrain appears contradictory to our finding that cortical OLs are derived from the medial dorsoventral boundary region. One possible explanation is that Wnt signaling is inhibited by endogenous factor(s) in this region. However, this seems unlikely because Axin2, a downstream Wnt target, is expressed in the medial boundary region [10]. Alternatively, an oligodendrogenesis-inducing factor(s) may overcome this Wnt activity.

Proteins of the FGF family are also good candidates for potential oligodendrogenesis-inducing factors. FGF-2 has important effects on the proliferation, migration, and differentiation of OPCs, and also affects the morphology and function of mature OLs [50-52]. FGF-2 also stimulates the generation of OLs from cultured cortical neural precursors of both the embryonic spinal cord and cerebral cortex [53-56]. Consistent with these in vitro studies, microinjection of FGF2 into the lateral ventricle of E13.5 mouse embryos increases the proliferation of neuroepithelial cells and the number of OPCs generated from the GE. In addition, ectopic generation of OPCs is observed in the dorsal cortex near the lateral and medial dorsoventral boundary [57], suggesting that FGF-2 overrides Wnt activity and induces the generation of OPCs from the medial dorsoventral boundary region (Fig. 6a). Further analysis of the interaction between Wnt and FGF signaling is needed to clarify these roles.

\section{Perspectives}

The cortico-striatal border has been shown to be a specialized region for the generation of cortical OLs in the adult mouse brain. This finding may contribute to future 
investigation into unknown mechanisms responsible for constructing the mammalian brain. During the period of early embryogenesis, neural precursor cells at the lateral dorsoventral boundary migrate out to the primordium of the basal telencephalic limbic system [58]. These neural precursor cells seem to produce neurons of the piriform cortex and amygdala during earlier embryonic stages, then switch their fate to produce cortical OLs during later embryonic and postnatal stages, although the molecular mechanism for this fate switch remains unknown. Here, the question arises: what is the function of neural precursor cells at the medial dorsoventral boundary? Given that the lateral domain of the cortico-striatal border is involved in neurogenesis of the limbic system, it is reasonable to hypothesize that the contralateral side has a similar purpose. This is an intriguing possibility because development of the limbic system remains largely unexplained. Moreover, since limbic structures regulate many fundamental traits such as emotions, memory, and drives, elucidating their development may provide us with clues to a better scientific understanding and potential future cures of psychiatric disorders.

\section{References}

1. Iglesias-Rozas JR, Garrosa M (2012) Glia with very few processes (oligodendroglia) by Pío Del Río-Hortega. Clin Neuropathol 31:440-459

2. Boulanger JJ, Messier C (2014) From precursors to myelinating oligodendrocytes: contribution of intrinsic and extrinsic factors to white matter plasticity in the adult brain. Neuroscience 269:343-366

3. Gage FH (2000) Mammalian neural stem cells. Science 287:1433-1438

4. Yamaguchi Y, Seki T, Imayoshi I, Tamamaki N, Hayashi Y, Tatebayashi Y, Hitoshi S (2016) Neural stem cells and neuro/ gliogenesis in the central nervous system: understanding the structural and functional plasticity of the developing, mature, and diseased brain. J Physiol Sci 66:197-206

5. Dawson MR, Polito A, Levine JM, Reynolds R (2003) NG2expressing glial progenitor cells: an abundant and widespread population of cycling cells in the adult rat CNS. Mol Cell Neurosci 24:476-488

6. Crawford AH, Stockley JH, Tripathi RB, Richardson WD, Franklin RJ (2014) Oligodendrocyte progenitors: adult stem cells of the central nervous system? Exp Neurol 260:50-55

7. Raff MC, Durand B, Gao FB (1998) Cell number control and timing in animal development: the oligodendrocyte cell lineage. Int J Dev Biol 42:263-267

8. Okano H, Temple S (2009) Cell types to order: temporal specification of CNS stem cells. Curr Opin Neurobiol 19:112-119

9. Alvarez-Buylla A, Garcia-Verdugo JM, Tramontin AD (2001) A unified hypothesis on the lineage of neural stem cells. Nat Rev Neurosci 2:287-293

10. Naruse M, Ishino Y, Kumar A, Ono K, Takebayashi H, Yamaguchi M, Ishizaki Y, Ikenaka K, Hitoshi S (2015) The dorsoventral boundary of the germinal zone is a specialized niche for the generation of cortical oligodendrocytes during a restricted temporal window. Cereb Cortex 26:2800-2810

11. Furusho M, Ono K, Takebayashi H, Masahira N, Kagawa T, Ikeda K, Ikenaka K (2006) Involvement of the Olig2 transcription factor in cholinergic neuron development of the basal forebrain. Dev Biol 293:348-357

12. Ono K, Takebayashi H, Ikeda K, Furusho M, Nishizawa T, Watanabe K, Ikenaka K (2008) Regional- and temporal-dependent changes in the differentiation of Olig2 progenitors in the forebrain, and the impact on astrocyte development in the dorsal pallium. Dev Biol 320:456-468

13. Masahira N, Takebayashi H, Ono K, Watanabe K, Ding L, Furusho M, Ogawa Y, Nabeshima Y, Alvarez-Buylla A, Shimizu K, Ikenaka K (2006) Olig2-positive progenitors in the embryonic spinal cord give rise not only to motoneurons and oligodendrocytes, but also to a subset of astrocytes and ependymal cells. Dev Biol 293:358-369

14. Mehler MF (2002) Mechanisms regulating lineage diversity during mammalian cerebral cortical neurogenesis and gliogenesis. Results Probl Cell Differ 39:27-52

15. Shimogori T, Banuchi V, Ng HY, Strauss JB, Grove EA (2004) Embryonic signaling centers expressing BMP, WNT and FGF proteins interact to pattern the cerebral cortex. Development 131:5639-5647

16. Liem KF Jr, Tremml G, Jessell TM (1997) A role for the roof plate and its resident TGFbeta-related proteins in neuronal patterning in the dorsal spinal cord. Cell 91:127-138

17. Grove EA, Tole S, Limon J, Yip L, Ragsdale CW (1998) The hem of the embryonic cerebral cortex is defined by the expression of multiple Wnt genes and is compromised in Gli3-deficient mice. Development 125:2315-2325

18. Muroyama Y, Fujihara M, Ikeya M, Kondoh H, Takada S (2002) Wnt signaling plays an essential role in neuronal specification of the dorsal spinal cord. Genes Dev 16:548-553

19. Chizhikov VV, Millen KJ (2005) Roof plate-dependent patterning of the vertebrate dorsal central nervous system. Dev Biol 277:287-295

20. Sousa VH, Fishell G (2010) Sonic hedgehog functions through dynamic changes in temporal competence in the developing forebrain. Curr Opin Genet Dev 20:391-399

21. Echelard Y, Epstein DJ, St-Jacques B, Shen L, Mohler J, McMahon JA, McMahon AP (1993) Sonic hedgehog, a member of a family of putative signaling molecules, is implicated in the regulation of CNS polarity. Cell 75:1417-1430

22. Fuccillo M, Rallu M, McMahon AP, Fishell G (2004) Temporal requirement for hedgehog signaling in ventral telencephalic patterning. Development 131:5031-5040

23. Yu K, McGlynn S, Matise MP (2013) Floor plate-derived sonic hedgehog regulates glial and ependymal cell fates in the developing spinal cord. Development 140:1594-1604

24. Jessell TM (2000) Neuronal specification in the spinal cord: inductive signals and transcriptional codes. Nat Rev Genet $1: 20-29$

25. Dessaud E, McMahon AP, Briscoe J (2008) Pattern formation in the vertebrate neural tube: a sonic hedgehog morphogen-regulated transcriptional network. Development 135:2489-2503

26. Zappone MV, Galli R, Catena R, Meani N, De Biasi S, Mattei E, Tiveron C, Vescovi AL, Lovell-Badge R, Ottolenghi S, Nicolis SK (2000) Sox 2 regulatory sequences direct expression of a $\beta$ geo transgene to telencephalic neural stem cells and precursors of the mouse embryo, revealing regionalization of gene expression in CNS stem cells. Development 127:2367-2382

27. Hitoshi S, Tropepe V, Sirard C, Ekker M, van der Kooy D (2002) Neural stem cell lineages are regionally specified, but not committed, within distinct compartments of the developing brain. Development 129:233-244 
28. Richardson WD, Smith HK, Sun T, Pringle NP, Hall A, Woodruff $\mathrm{R}$ (2000) Oligodendrocyte lineage and the motor neuron connection. Glia 29:136-142

29. He L, Lu QR (2013) Coordinated control of oligodendrocyte development by extrinsic and intrinsic signaling cues. Neurosci Bull 29:129-143

30. Cai J, Qi Y, Hu X, Tan M, Liu Z, Zhang J, Li Q, Sander M, Qiu M (2005) Generation of oligodendrocyte precursor cells from mouse dorsal spinal cord independent of Nkx6 regulation and Shh signaling. Neuron 45:41-53

31. Vallstedt A, Klos JM, Ericson J (2005) Multiple dorsoventral origins of oligodendrocyte generation in the spinal cord and hindbrain. Neuron 45:55-67

32. Takebayashi H, Yoshida S, Sugimori M, Kosako H, Kominami R, Nakafuku M, Nabeshima Y (2000) Dynamic expression of basic helix-loop-helix Olig family members: implication of Olig2 in neuron and oligodendrocyte differentiation and identification of a new member, Olig3. Mech Dev 99:143-148

33. Lu QR, Yuk D, Alberta JA, Zhu Z, Pawlitzky I, Chan J, McMahon AP, Stiles CD, Rowitch DH (2000) Sonic hedgehogregulated oligodendrocyte lineage genes encoding bHLH proteins in the mammalian central nervous system. Neuron 25:317-329

34. Zhou Q, Wang S, Anderson DJ (2000) Identification of a novel family of oligodendrocyte lineage-specific basic helix-loop-helix transcription factors. Neuron 25:331-343

35. Takebayashi H, Nabeshima Y, Yoshida S, Chisaka O, Ikenaka K, Nabeshima Y (2002) The basic helix-loop-helix factor olig2 is essential for the development of motoneuron and oligodendrocyte lineages. Curr Biol 12:1157-1163

36. Lu QR, Sun T, Zhu Z, Ma N, Garcia M, Stiles CD, Rowitch DH (2002) Common developmental requirement for Olig function indicates a motor neuron/oligodendrocyte connection. Cell 109:75-86

37. Li H, de Faria JP, Andrew P, Nitarska J, Richardson WD (2011) Phosphorylation regulates OLIG2 cofactor choice and the motor neuron-oligodendrocyte fate switch. Neuron 69:918-929

38. Fogarty M, Richardson WD, Kessaris N (2005) A subset of oligodendrocytes generated from radial glia in the dorsal spinal cord. Development 132:1951-1959

39. Tripathi RB, Clarke LE, Burzomato V, Kessaris N, Anderson PN, Attwell D, Richardson WD (2011) Dorsally and ventrally derived oligodendrocytes have similar electrical properties but myelinate preferred tracts. J Neurosci 31:6809-6819

40. Bulfone A, Puelles L, Porteus MH, Frohman MA, Martin GR, Rubenstein JL (1993) Spatially restricted expression of $D l x-1$, $D l x-2$ (Tes-1), Gbx-2, and Wnt-3 in the embryonic day 12.5 mouse forebrain defines potential transverse and longitudinal segmental boundaries. J Neurosci 13:3155-3172

41. Woodruff RH, Tekki-Kessaris N, Stiles CD, Rowitch DH, Richardson WD (2001) Oligodendrocyte development in the spinal cord and telencephalon: common themes and new perspectives. Int J Dev Neurosci 19:379-385

42. Kessaris N, Pringle N, Richardson WD (2001) Ventral neurogenesis and the neuron-glial switch. Neuron 31:677-680

43. Tekki-Kessaris N, Woodruff R, Hall AC, Gaffield W, Kimura S, Stiles CD, Rowitch DH, Richardson WD (2001) Hedgehog- dependent oligodendrocyte lineage specification in the telencephalon. Development 128:2545-2554

44. Parras CM, Hunt C, Sugimori M, Nakafuku M, Rowitch D, Guillemot F (2007) The proneural gene Mash1 specifies an early population of telencephalic oligodendrocytes. J Neurosci 27:4233-4242

45. Kessaris N, Fogarty M, Iannarelli P, Grist M, Wegner M, Richardson WD (2006) Competing waves of oligodendrocytes in the forebrain and postnatal elimination of an embryonic lineage. Nat Neurosci 9:173-179

46. Nakahira E, Kagawa T, Shimizu T, Goulding MD, Ikenaka K (2006) Direct evidence that ventral forebrain cells migrate to the cortex and contribute to the generation of cortical myelinating oligodendrocytes. Dev Biol 291:123-131

47. Shimizu T, Kagawa T, Wada T, Muroyama Y, Takada S, Ikenaka $\mathrm{K}$ (2005) Wnt signaling controls the timing of oligodendrocyte development in the spinal cord. Dev Biol 282:397-410

48. Langseth AJ, Munji RN, Choe Y, Huynh T, Pozniak CD, Pleasure SJ (2010) Wnts influence the timing and efficiency of oligodendrocyte precursor cell generation in the telencephalon. J Neurosci 30:13367-13372

49. Fancy SP, Baranzini SE, Zhao C, Yuk DI, Irvine KA, Kaing S, Sanai N, Franklin RJ, Rowitch DH (2009) Dysregulation of the Wnt pathway inhibits timely myelination and remyelination in the mammalian CNS. Genes Dev 23:1571-1585

50. Fortin D, Rom E, Sun H, Yayon A, Bansal R (2005) Distinct fibroblast growth factor (FGF)/FGF receptor signaling pairs initiate diverse cellular responses in the oligodendrocyte lineage. J Neurosci 25:7470-7479

51. Bansal R (2002) Fibroblast growth factors and their receptors in oligodendrocyte development: implications for demyelination and remyelination. Dev Neurosci 24:35-46

52. Bansal R, Pfeiffer SE (1997) FGF-2 converts mature oligodendrocytes to a novel phenotype. J Neurosci Res 50:215-228

53. Qian X, Davis AA, Goderie SK, Temple S (1997) FGF2 concentration regulates the generation of neurons and glia from multipotent cortical stem cells. Neuron 18:81-93

54. Gabay L, Lowell S, Rubin LL, Anderson DJ (2003) Deregulation of dorsoventral patterning by FGF confers trilineage differentiation capacity on CNS stem cells in vitro. Neuron 40:485-499

55. Chandran S, Kato H, Gerreli D, Compston A, Svendsen CN, Allen ND (2003) FGF-dependent generation of oligodendrocytes by a hedgehog-independent pathway. Development 130:6599-6609

56. Kessaris N, Jamen F, Rubin LL, Richardson WD (2004) Cooperation between sonic hedgehog and fibroblast growth factor/ MAPK signalling pathways in neocortical precursors. Development 131:1289-1298

57. Naruse M, Nakahira E, Miyata T, Hitoshi S, Ikenaka K, Bansal R (2006) Induction of oligodendrocyte progenitors in dorsal forebrain by intraventricular microinjection of FGF-2. Dev Biol 297:262-273

58. Carney RS, Alfonso TB, Cohen D, Dai H, Nery S, Stoica B, Slotkin J, Bregman BS, Fishell G, Corbin JG (2006) Cell migration along the lateral cortical stream to the developing basal telencephalic limbic system. J Neurosci 26:11562-11574 